\title{
Second Order Statistics of Non-Isotropic Mobile-to-Mobile Ricean Fading Channels
}

\author{
Xiang Cheng ${ }^{\dagger}$, Cheng-Xiang Wang ${ }^{\dagger}$, David I. Laurenson ${ }^{\dagger \dagger}$, and Athanasios V. Vasilakos ${ }^{\dagger \dagger}$

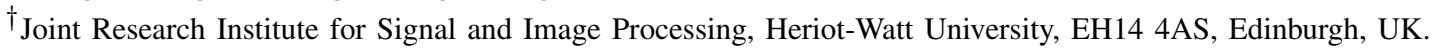 \\ ${ }^{\dagger \dagger}$ Joint Research Institute for Signal and Image Processing, University of Edinburgh, EH9 3JL, Edinburgh, UK. \\ ${ }^{\dagger \dagger}$ Department of Computer and Telecommunications Engineering, University of Western Macedonia, GR 50100 Kozani, Greece. \\ Email: xc48@hw.ac.uk, cheng-xiang.wang@hw.ac.uk, dave.laurenson@ed.ac.uk, vasilako@ath.forthnet.gr
}

\begin{abstract}
This paper develops a generic geometry-based stochastic model for mobile-to-mobile (M2M) Ricean fading channels. From the generic model, the level crossing rate (LCR) and average fade duration (AFD) are derived. Based on the derived expressions, we for the first time investigate the LCR and AFD for M2M channels with different vehicular traffic densities (VTDs). Excellent agreement is achieved between the theoretical results and measured data, demonstrating the utility of the proposed model.
\end{abstract}

\section{INTRODUCTION}

In mobile communications with time-variant fading, the LCR and AFD are two important second order statistics, which characterize some aspects of the dynamic temporal behavior of envelope fluctuations. The LCR and AFD can be used to investigate the disturbing effects of signal fades and consequently appear in a variety of applications, such as the interleaver optimization and design of adaptive modulation schemes.

In recent years, M2M communications have received increasing attention due to some new applications, such as wireless mobile ad hoc networks, relay-based cellular networks, and dedicated short range communications (DSRC) for intelligent transportation systems (IEEE 802.11p). Such M2M systems consider that both the transmitter (Tx) and receiver (Rx) are in motion and equipped with low elevation antennas. To successfully analyze and design M2M systems, it is necessary to have detailed knowledge of the multipath fading channel and its statistical properties. Some important characteristics of M2M channels, such as correlation properties and Doppler power spectral densities (PSDs), have been explored in many papers. Akki and Haber [1] were among the first to study the time correlations and corresponding Doppler PSDs for isotropic single-input single-output (SISO) M2M Rayleigh fading channels. Space-time correlations and corresponding Doppler PSDs for non-isotropic multiple-input multiple-output (MIMO) M2M Ricean fading channels were analyzed in [2][4]. More recently, in [5] and [6], we investigated in more detail the space-time-frequency correlations and corresponding Doppler PSDs for non-isotropic MIMO M2M Ricean fading channels. However, to the best of the authors' knowledge, although the LCR and AFD are of extreme importance for a M2M channel, only [7] and [8] investigated the LCR and AFD for M2M channels. In [7], the LCR and AFD for isotropic M2M Rayleigh fading channels in two-dimensional
(2D) macro-cell scenarios were studied. In [8], the authors investigated the LCR and AFD for non-isotropic M2M Ricean fading channels in three-dimensional (3D) macro- and microcells scenarios as well as giving the corresponding experimental validation. However, none of these two papers investigated the LCR and AFD in the case of pico-cell scenarios, which are currently receiving more and more attention in $\mathrm{M} 2 \mathrm{M}$ systems for DSRC. Considering the unique feature of M2M communication environments, the impact of VTD on channel characteristics in micro- and pico-cell scenarios cannot be neglected as in macro-cell scenarios. However, this impact on the LCR and AFD has not been investigated yet.

To fill the aforementioned gaps, our first task is to have a M2M channel model that has the ability to mimic pico-cell scenarios and take the impact of VTD into account. To this end, following the same structure of our MIMO M2M model in [6], we first develop a new generic 2D SISO M2M geometrybased stochastic model, which is suitable for a wide variety of outdoor scenarios, i.e., macro-, micro-, as well as pico-cell scenarios, and has the ability to consider the impact of VTD on channel characteristics. This model employs a combined two-ring model and ellipse model, where the received signal is constructed as a sum of the line-of-sight (LoS), single-, and double-bounced rays with different energies. From the presented model, we derive the LCR and AFD for nonisotropic M2M Ricean fading channels. Therefore, the derived analytical expressions allow us to analyze the LCR and AFD in a wide variety of $\mathrm{M} 2 \mathrm{M}$ scenarios, especially for pico-cell scenarios, and to study the impact of VTD on the LCR and AFD. Our analysis shows several flaws in the derivation and investigation of the LCR and AFD in [8], revealing some easily neglected but significant issues. Therefore, we clearly address these issues in Section III. Finally, comparisons of the obtained theoretical LCR and AFD against measurement data in [9] are presented. Excellent agreement between them demonstrates the utility of the proposed model.

\section{A Generic Model For M2M Channels}

Let us now consider a narrowband single-user SISO M2M communication system. Both the $\mathrm{Tx}$ and $\mathrm{Rx}$ are in motion and equipped with low elevation antennas. The propagation scenario is characterized by a non-isotropic scattering with possibly a LoS component between the Tx and Rx. 
Fig. 1 illustrates the geometry of the SISO M2M model, which is the combination of a LoS component, a single- and double-bounce two-ring model and a single-bounce ellipse model. The two-ring model defines two rings of effective scatterers, one around the Tx and the other around the Rx. Suppose there are $N_{1}$ effective scatterers around the Tx lying on a ring of radius $R_{T}$ and the $n_{1}$ th $\left(n_{1}=1, \ldots, N_{1}\right)$ effective scatterer is denoted by $s^{\left(n_{1}\right)}$. Similarly, assume there are $N_{2}$ effective scatterers around the Rx lying on a ring of radius $R_{R}$ and the $n_{2}$ th $\left(n_{2}=1, \ldots, N_{2}\right)$ effective scatterer is denoted by $s^{\left(n_{2}\right)}$. For the ellipse model, $N_{3}$ effective scatterers lie on an ellipse with the Tx and Rx located at the foci. The semi-major axis of the ellipse and the $n_{3}$ th $\left(n_{3}=1, \ldots, N_{3}\right)$ effective scatterer are denoted by $a$ and $s^{\left(n_{3}\right)}$, respectively. The distance between the Tx and Rx is $D=2 f$ with $f$ denoting the half length of the distance between the two focal points of the ellipse. The Tx and Rx move with speeds $v_{T}$ and $v_{R}$ in directions determined by the angles of motion $\gamma_{T}$ and $\gamma_{R}$, respectively. The symbol $\phi_{R_{q}}^{L o S}$ denotes the angle of arrival (AoA) of a LoS path. The AoA of the wave traveling from the effective scatterer $s^{\left(n_{i}\right)}(i \in\{1,2,3\})$ toward the $\mathrm{Rx}$ is denoted by $\phi_{R}^{\left(n_{i}\right)}$. The angle of departure (AoD) of the wave that impinges on the effective scatterer $s^{\left(n_{i}\right)}$ is designated by $\phi_{T}^{\left(n_{i}\right)}$.

From the above model, the received complex impulse response is a superposition of the $\mathrm{LoS}$ component and diffuse component, which consists of single-, and double-bounced rays, and can be expressed as

$$
h(t)=h^{L o S}(t)+h^{D I F}(t)=h^{L o S}(t)+h^{S B}(t)+h^{D B}(t)
$$

where

$$
\begin{aligned}
& h^{L o S}(t)=\sqrt{\frac{K \Omega}{K+1}} e^{j 2 \pi f_{T_{\max }} t \cos \left(\pi-\phi_{R}^{L o S}+\gamma_{T}\right)} \\
& \times e^{j 2 \pi f_{R_{\text {max }}} t \cos \left(\phi_{R}^{L o S}-\gamma_{R}\right)} \\
& h^{S B}(t)=\sum_{i=1}^{I} h^{S B_{i}}(t)=\sum_{i=1}^{I} \sqrt{\frac{\eta_{S B_{i}} \Omega}{K+1}} \lim _{N_{i} \rightarrow \infty} \sum_{n_{i}=1}^{N_{i}} \frac{1}{\sqrt{N_{i}}} e^{j \psi_{n_{i}}} \\
& \times e^{j\left[2 \pi f_{T_{\max }} t \cos \left(\phi_{T}^{\left(n_{i}\right)}-\gamma_{T}\right)+2 \pi f_{R_{\max }} t \cos \left(\phi_{R}^{\left(n_{i}\right)}-\gamma_{R}\right)\right]} \\
& h^{D B}(t)=\sqrt{\frac{\eta_{D B} \Omega}{K+1}} \lim _{N_{1}, N_{2} \rightarrow \infty_{n_{1}, n_{2}=1}} \sum_{N_{1}, N_{2}}^{{\sqrt{N_{1} N_{2}}}} e^{j \psi_{n_{1}, n_{2}}} \\
& \times e^{j\left[2 \pi f_{T_{\max }} t \cos \left(\phi_{T}^{\left(n_{1}\right)}-\gamma_{T}\right)+2 \pi f_{R_{\max }} t \cos \left(\phi_{R}^{\left(n_{2}\right)}-\gamma_{R}\right)\right]}
\end{aligned}
$$

where $i \in\{1,2, \ldots, I\}$ with $I=3$. The symbol $K$ designates the Ricean factor (ratio of the LoS component power to the diffuse component power) and $\Omega$ denotes the total power. Parameters $\eta_{S B_{i}}$ and $\eta_{D B}$ specify how much the single- and double-bounced rays contribute to the total scattered power $\Omega /(K+1)$, respectively. Note that these energy-related parameters satisfy $\sum_{i=1}^{I} \eta_{S B_{i}}+\eta_{D B}=1$. The phases $\psi_{n_{i}}$ and $\psi_{n_{i}, n_{j}}$ are independent and identically distributed (i.i.d.) random variables with uniform distributions over $[-\pi, \pi), f_{T_{\max }}=v_{T} / \lambda$ and $f_{R_{\max }}=v_{R} / \lambda$ are the maximum Doppler frequencies with respect to the Tx and Rx, respectively, and $\lambda$ is the carrier wavelength. Following the same approach on how to adjust the important model parameters (distance $D$, the energy-related parameters $\eta_{S B_{i}}$ and $\eta_{D B}$, and the Ricean factor $K$ ) in [6], we can make the proposed model suitable for a wide variety of scenarios, i.e., macro-cell, micro-cell, and pico-cell scenarios. Note that the assumption $D \gg \max \left\{R_{T}, R_{R}\right\}$ widely used in macro- and micro-cell scenarios is not applicable to picocell scenarios. To allow our model to have the ability to consider the impact of VTD, we use the two-ring model to describe the moving scatterers around the Tx and $\mathrm{Rx}$ and utilize the ellipse model to depict the stationary scatterers located on the roadside. Note that the $\mathrm{AoD} \phi_{T}^{\left(n_{i}\right)}$ and AoA $\phi_{R}^{\left(n_{i}\right)}$ are independent random variables for double-bounced rays, while they are interdependent for single-bounced rays. By using the results in [6], we can express the general relationships between the AoD and AoA for the single-bounced two-ring model as $\phi_{R}^{\left(n_{1}\right)}=\arcsin \left[R_{T} \sin \phi_{T}^{\left(n_{1}\right)} /\left(R_{T}^{2}+D^{2}-\right.\right.$ $\left.\left.2 R_{T} D \cos \phi_{T}^{\left(n_{1}\right)}\right)^{-1 / 2}\right]$ and $\phi_{T}^{\left(n_{2}\right)}=\arcsin \left[R_{R} \sin \phi_{R}^{\left(n_{2}\right)} /\left(R_{R}^{2}+\right.\right.$ $\left.\left.D^{2}+2 R_{R} D \cos \phi_{R}^{\left(n_{2}\right)}\right)^{-1 / 2}\right]$ and for ellipse model as $\phi_{T}^{\left(n_{3}\right)}=$ $\arcsin \left[b^{2} \sin \phi_{R}^{\left(n_{3}\right)} /\left(a^{2}+f^{2}+2 a f \cos \phi_{R}^{\left(n_{3}\right)}\right)\right]$, where $b$ denotes the semi-minor axis of the ellipse and the equality $a^{2}=b^{2}+f^{2}$ holds. These exact relationships are sufficiently general and thus suitable for a wide variety of scenarios. Moreover, based on the assumption $D \gg \max \left\{R_{T}, R_{R}\right\}$ in macro- and microcell scenarios, the exact relationships for the single-bounced two-ring model can reduce to the widely used approximate relationships $\phi_{R}^{\left(n_{1}\right)} \approx \pi-\Delta_{T} \sin \phi_{T}^{\left(n_{1}\right)}$ and $\phi_{T}^{\left(n_{2}\right)} \approx \Delta_{R} \sin \phi_{R}^{\left(n_{2}\right)}$ with $\Delta_{T} \approx R_{T} / D$ and $\Delta_{R} \approx R_{R} / D$.

Since we assume that the number of effective scatterers in the model tends to infinity (as shown in (2)-(4)), the discrete $\mathrm{AoA}, \phi_{R}^{\left(n_{i}\right)}$, and $\mathrm{AoD}, \phi_{T}^{\left(n_{i}\right)}$, can be replaced by continuous random variables $\phi_{R}^{S B_{i}}$ and $\phi_{T}^{S B_{i}}$, respectively. In the literature, many different scatterer distributions have been proposed to characterize the AoD $\phi_{T}^{S B_{i}}$ and AoA $\phi_{R}^{S B_{i}}$, such as the uniform, Gaussian, wrapped Gaussian, and cardioid PDFs. In this paper, the von Mises PDF [13] is used, which can approximate all the above mentioned PDFs. The von Mises PDF is defined as $f(\phi) \triangleq \exp [k \cos (\phi-\mu)] / 2 \pi I_{0}(k)$, where $\phi \in[-\pi, \pi), I_{0}(\cdot)$ is the zeroth-order modified Bessel function of the first kind, $\mu \in[-\pi, \pi)$ accounts for the mean value of the angle $\phi$, and $k(k \geq 0)$ is a real-valued parameter that controls the angle spread of the angle $\phi$.

\section{SECond ORder Statistics}

In this section, based on the proposed model in (1)-(4), we will derive the LCR and AFD for a non-isotropic scattering environment. The LCR, $L_{\xi}\left(r_{l}\right)$, is by definition the average number of times per second that the signal envelope, $\xi(t)=$ $|h(t)|$, crosses a specified level $r_{l}$ with positive/negative slope. Using the traditional PDF-based method [11], we derive the general expression of LCR for M2M Ricean fading channels as

$$
\begin{aligned}
& L_{\xi}\left(r_{l}\right)=\frac{2 r_{l} e^{-K-(K+1) r_{l}^{2}}}{\pi^{3 / 2}} \sqrt{\frac{B(K+1)}{b_{0}}} \int_{0}^{\pi / 2} \cosh \left(2 \sqrt{K(K+1)} r_{l} \cos \theta\right) \\
& \times\left[e^{-\left(\frac{\varsigma \zeta \sin \theta}{\sqrt{2 B}}\right)^{2}}+\sqrt{\frac{\pi}{2 B}} \varsigma \zeta \sin \theta \cdot \operatorname{erf}\left(\frac{\varsigma \zeta \sin \theta}{\sqrt{2 B}}\right)\right] d \theta
\end{aligned}
$$


where $\cosh (\cdot)$ is the hyperbolic cosine, $\operatorname{erf}(\cdot)$ denotes the error function, and parameters $B, \zeta$, and $\varsigma$ are $B=b_{2}-b_{1} / b_{0}$, $\zeta=\sqrt{K \Omega /(K+1)}$, and $\varsigma=E+b_{1} / b_{0}$, respectively, with $E=2 \pi f_{T_{\max }} \cos \left(\pi-\phi_{R}^{L o S}+\gamma_{T}\right)+2 \pi f_{R_{\max }} \cos \left(\phi_{R}^{L o S}-\gamma_{R}\right)$. Finally, the key parameter $b_{m}(m=0,1,2)$ is defined as

$$
b_{m}=\left.\frac{d^{m} \rho_{h^{D I F}(\tau)}}{2 j^{m} d \tau^{m}}\right|_{\tau=0}
$$

where $j^{2}=-1, \rho_{h^{D I F}}(\tau)=\mathbf{E}\left[h^{D I F}(t) h^{D I F *}(t-\tau)\right] / \Omega$ is the normalized time autocorrelation function (ACF) of the complex impulse response of diffuse component $h^{D I F}(t),(\cdot)^{*}$ denotes the complex conjugate operation, and $\mathbf{E}[\cdot]$ designates the statistical expectation operator. Note that if we consider only one terminal (Tx or Rx) is in motion and decompose the complex impulse response into the in-phase and quadrature components, (5) has the same expression as (29) in [12].

Before deriving the key parameter $b_{m}(m=0,1,2, \ldots$,$) of$ the LCR in (5), we would like to first clarify the relationship of different LCR expressions for Ricean fading channels, i.e., (5) in this paper, (6) in [8], and (2.99) in [10]. These expressions were given separately in different papers and should be used carefully for different propagation environments. It is obvious that (5) is the general expression and can reduce to (6) in [8] by setting $E=0$. If we further assume $b_{1}=0$ in (5) (it follows that $\varsigma=0$ ), we can obtain the closed-form LCR expression in (2.99) in [10]. We can therefore conclude that for a non-isotropic scattering environment $\left(b_{1} \neq 0\right)$ with a timedependent LoS component $(E \neq 0)$, we can only choose (5) as the LCR expression. While for a non-isotropic scattering environment with a time-independent $\operatorname{LoS}$ component $(E=0)$, either (5) here with $E=0$ or (6) in [8] can be used. The LCR expression in (2.99) in [10] is suitable only for an isotropic scattering environment $\left(b_{1}=0\right)$ with a time-independent LoS component. Since the investigated environment in this paper is a non-isotropic scattering environment with a time-dependent LoS component (as shown in (2)), (5) is used here as the LCR expression. In [8], the authors misapplied (6) as the expression to study the LCR for exactly the same environment as in this paper.

Based on the presented model in Section II, we now derive the key parameter $b_{m}$. By substituting (1)-(4) into (6) and setting $m=0$, the parameter $b_{0}$ becomes

$$
b_{0}=b_{0}^{S B_{1}}+b_{0}^{S B_{2}}+b_{0}^{S B_{3}}+b_{0}^{D B}=\frac{1}{2(K+1)}
$$

where $b_{0}^{S B_{1}}=\frac{\eta_{S B_{1}}}{2(K+1)}, b_{0}^{S B_{2}}=\frac{\eta_{S B_{2}}}{2(K+1)}, b_{0}^{S B_{3}}=\frac{\eta_{S B_{3}}}{2(K+1)}$, and $b_{0}^{D B}=\frac{\eta_{D B}}{2(K+1)}$. Note that (7) corrects the expression of $b_{0}$ in (10) in [8], where $b_{0}=\frac{1}{K+1}$. Similarly, by substituting (1)-(4) into (6), we have the parameters $b_{1}$ and $b_{2}$ as

$$
b_{n}=b_{n}^{S B_{1}}+b_{n}^{S B_{2}}+b_{n}^{S B_{3}}+b_{n}^{D B}, \quad n=1,2 .
$$

By considering the von Mise PDF for two-ring scatterers, as $f\left(\phi_{T}^{S B_{1}}\right)=\exp \left[k_{T}^{T R} \cos \left(\phi_{T}^{S B_{1}}-\mu_{T}^{T R}\right)\right] / 2 \pi I_{0}\left(k_{T}^{T R}\right)$ for the ring of scatterers around the Tx and $f\left(\phi_{R}^{S B_{2}}\right)=\exp \left[k_{R}^{T R}\right.$ $\left.\times \cos \left(\phi_{R}^{S B_{2}}-\mu_{R}^{T R}\right)\right] / 2 \pi I_{0}\left(k_{R}^{T R}\right)$ for the ring of scatterers around the Rx, the parameters $b_{n}^{S B_{1(2)}}$ can be expressed as

$$
\begin{aligned}
& b_{1}^{S B_{1(2)}}=b_{0}^{S B_{1(2)}} \int_{-\pi}^{\pi} \frac{e^{k_{T(R)}^{T R} \cos \left(\phi_{T(R)}^{S B_{1(2)}}-\mu_{T(R)}^{T R}\right)}}{I_{0}\left(k_{T(R)}^{T R}\right)}\left[f_{T_{\max }}\right. \\
& \left.\times \cos \left(\phi_{T}^{S B_{1(2)}}-\gamma_{T}\right)+f_{R_{\max }} \cos \left(\phi_{R}^{S B_{1(2)}}-\gamma_{R}\right)\right] d \phi_{T(R)}^{S B_{1(2)}}
\end{aligned}
$$

$$
\begin{aligned}
& b_{2}^{S B_{1(2)}}=b_{0}^{S B_{1(2)}} \int_{-\pi}^{\pi} \frac{2 \pi e^{k_{T(R)}^{T R} \cos \left(\phi_{T(R)}^{S B_{1(2)}}-\mu_{T(R)}^{T R}\right)}}{I_{0}\left(k_{T(R)}^{T R}\right)}\left[f_{T_{\max }}\right. \\
& \left.\times \cos \left(\phi_{T}^{S B_{1(2)}}-\gamma_{T}\right)+f_{R_{\max }} \cos \left(\phi_{R}^{S B_{1(2)}}-\gamma_{R}\right)\right]^{2} d \phi_{T(R)}^{S B_{1(2)}} .
\end{aligned}
$$

By following the general relationship between the AoD and AoA of the single-bounced two-ring model given in Section II, the parameters $\phi_{R}^{S B_{1}}$ and $\phi_{T}^{S B_{2}}$ in (9) and (10) can be expressed by $\phi_{T}^{S B_{1}}$ and $\phi_{R}^{S B_{2}}$, respectively. Similarly, by considering the von Mises PDF for ellipse scatterers as $f\left(\phi_{R}^{S B_{3}}\right)=\exp \left[k_{R}^{E R} \cos \left(\phi_{R}^{S B_{3}}-\mu_{R}^{E R}\right)\right] / 2 \pi I_{0}\left(k_{R}^{E R}\right)$, the parameters $b_{n}^{S B_{3}}$ can be obtained as

$$
\begin{aligned}
b_{1}^{S B_{3}} & =b_{0}^{S B_{3}} \int_{-\pi}^{\pi} \frac{e^{k_{R}^{E R} \cos \left(\phi_{R}^{S B_{3}}-\mu_{R}^{E R}\right)}}{I_{0}\left(k_{R}^{E R}\right)}\left[f_{T_{\max }} \cos \left(\phi_{T}^{S B_{3}}-\gamma_{T}\right)\right. \\
& \left.+f_{R_{\max }} \cos \left(\phi_{R}^{S B_{3}}-\gamma_{R}\right)\right] d \phi_{R}^{S B_{3}} \\
b_{2}^{S B_{3}} & =b_{0}^{S B_{3}} \int_{-\pi}^{\pi} \frac{2 \pi e^{k_{R}^{E R} \cos \left(\phi_{R}^{S B_{3}}-\mu_{R}^{E R}\right)}}{I_{0}\left(k_{R}^{E R}\right)}\left[f_{T_{\max }} \cos \left(\phi_{T}^{S B_{3}}-\gamma_{T}\right)\right. \\
& \left.+f_{R_{\max }} \cos \left(\phi_{R}^{S B_{3}}-\gamma_{R}\right)\right]^{2} d \phi_{R}^{S B_{3}}
\end{aligned}
$$

By following the general relationship of the ellipse model shown in Section II, the parameter $\phi_{T}^{S B_{3}}$ in (11) and (12) can be expressed by $\phi_{R}^{S B_{3}}$. Considering the von Mise PDF for two-ring scatterers again as well as applying trigonometric transformations, product rule for differentiation, and the following equalities $\int_{-\pi}^{\pi} e^{a \sin c+b \cos c} d c=2 \pi I_{0}\left(\sqrt{a^{2}+b^{2}}\right)$, $d I_{V}(z) / d z=\left[I_{V-1}(z)+I_{V+1}(z)\right] / 2$, and $z I_{V-1}(z)-$ $z I_{V+1}(z)=(V+1) I_{V}(z)$ [14], where $I_{V}(\cdot)$ is the $V^{\text {th }}$, order modified Bessel function of the first kind, we can get the closed-form expressions of parameters $b_{n}^{D B}$ as

$$
\begin{gathered}
b_{1}^{D B}=b_{0}^{D B}\left[\frac{2 \pi f_{T_{\max }} \cos \left(\gamma_{T}-\mu_{T}^{T R}\right) I_{1}\left(k_{T}^{T R}\right)}{I_{0}\left(k_{T}^{T R}\right)}\right. \\
\left.+\frac{2 \pi f_{R_{\max }} \cos \left(\gamma_{R}-\mu_{R}^{T R}\right) I_{1}\left(k_{R}^{T R}\right)}{I_{0}\left(k_{R}^{T R}\right)}\right] \\
b_{2}^{D B}=b_{0}^{D B}\left[4 \pi^{2} f_{T_{\max }}^{2} \frac{1+\cos \left(2\left(\gamma_{T}-\mu_{T}^{T R}\right)\right) I_{2}\left(k_{T}^{T R}\right)}{2 I_{0}\left(k_{T}^{T R}\right)}+\right. \\
4 \pi^{2} f_{R_{\max }}^{2} \frac{1+\cos \left(2\left(\gamma_{R}-\mu_{R}^{T R}\right)\right) I_{2}\left(k_{R}^{T R}\right)}{2 I_{0}\left(k_{R}^{T R}\right)}+8 \pi^{2} f_{T_{\max }} f_{R_{\max }} \\
\left.\times \cos \left(\gamma_{T}-\mu_{T}^{T R}\right) \cos \left(\gamma_{R}-\mu_{R}^{T R}\right) \frac{I_{1}\left(k_{T}^{T R}\right) I_{1}\left(k_{R}^{T R}\right)}{I_{0}\left(k_{T}^{T R}\right) I_{0}\left(k_{R}^{T R}\right)}\right] .
\end{gathered}
$$

Numerical integration methods are needed to evaluate the integrals in (9)-(12). However, for macro- and micro-cells scenarios, following the approximate relationships shown in 
Section II instead of the general ones, we can express the parameters $\phi_{R}^{S B_{1}}$ and $\phi_{T}^{S B_{2}}$ in (9) and (10) as $\phi_{R}^{S B_{1}} \approx \pi-$ $\Delta_{T} \sin \phi_{T}^{S B_{1}}$ and $\phi_{T}^{S B_{2}} \approx \Delta_{R} \sin \phi_{R}^{S B_{2}}$, respectively. In such a case, by applying trigonometric transformations and the equality $\int_{-\pi}^{\pi} e^{-j V \theta+z \sin \theta} d \theta=2 \pi(-j)^{V} I_{V}(z)[14]$, (9) and (10) become, respectively, the following closed-form expressions as $b_{1}^{S B_{1(2)}=} b_{0}^{S B_{1(2)}}\left[2 \pi W_{T(R)} \frac{I_{1}\left(k_{T(R)}^{T R}\right)}{I_{0}\left(k_{T(R)}^{T R}\right)} \mp 2 \pi f_{R(T)_{\max }} \cos \gamma_{R(T)}\right]$

$$
\begin{aligned}
b_{2}^{S B_{1(2)}}=b_{0}^{S B_{1(2)}}\left[2 \pi^{2} X_{T(R)}\right. & \mp 4 \pi^{2} Y_{T(R)} \frac{I_{1}\left(k_{T(R)}^{T R}\right)}{I_{0}\left(k_{T(R)}^{T R}\right)} \\
& \left.+2 \pi^{2} Z_{T(R)} \frac{I_{2}\left(k_{T(R)}^{T R}\right)}{I_{0}\left(k_{T(R)}^{T R}\right)}\right]
\end{aligned}
$$

where parameters $W_{T(R)}, X_{T(R)}, Y_{T(R)}$, and $Z_{T(R)}$ are $W_{T(R)}=f_{T(R)_{\max }} \cos \left(\gamma_{T(R)}-\mu_{T(R)}^{T R}\right)+f_{R(T)_{\max }} \Delta_{T(R)}$ $\times \sin \mu_{T(R)}^{T R} \sin \gamma_{R(T)}, X_{T(R)}=2 f_{R(T)_{\max }}^{2} \cos ^{2} \gamma_{R(T)}+\Delta_{T(R)}^{2}$ $\times f_{R(T)_{\max }}^{2} \sin ^{2} \gamma_{R(T)}+f_{T(R)_{\max }}^{2}+2 f_{T_{\max }} f_{R_{\max }} \Delta_{T(R)} \sin \gamma_{T}$ $\times \sin \gamma_{R}, \quad Y_{T(R)}=2 f_{T_{\text {max }}} f_{R_{\text {max }}} \cos \gamma_{R(T)} \cos \left(\gamma_{T(R)}-\mu_{T(R)}^{T R}\right)+$ $f_{R(T)_{\text {max }}}^{2} \Delta_{T(R)} \sin \left(2 \gamma_{R(T)}\right) \sin \mu_{T(R)}^{T R}$, and $Z_{T(R)}=f_{T(R)_{\max }}^{2}$ $\times \cos \left(2\left(\gamma_{T(R)}-\mu_{T(R)}^{T R}\right)\right)-f_{R(T)_{\max }}^{2} \Delta_{T(R)}^{2} \sin ^{2} \gamma_{R(T)} \cos \left(2 \mu_{T(R)}^{T R}\right)$ $-2 f_{T_{\text {max }}} f_{R_{\max }} \Delta_{T(R)} \sin \gamma_{R(T)} \sin \left(\gamma_{T(R)}-2 \mu_{T(R)}^{T R}\right)$, respectively. Note that the expressions of parameters $b_{n}$ in (9)-(14) make the LCR expression in (5) suitable for a wider variety of scenarios, while the expressions in (15) and (16) limit the application of (5) to only macro- and microcell scenarios. There are big differences between the above derived parameters $b_{n}^{S B_{1(2)}}$ in (15) and (16) and the derived parameters $b_{n}^{S B_{1(2)}}$ in (19) and (21) with $\beta_{T(R)}=\Delta_{H}=0$ (i.e., consider incoming signals only from the azimuthal plane (a 2D scattering environment)) in [8]. To determine which closed-form expressions of $b_{n}^{S B_{1(2)}}$ are correct, in Fig. 2 (a) we compare the LCRs having the same expression (5) but with different parameters $\left.b_{n}^{S B_{1(2)}}: 1\right) L_{\xi-C}^{T R}\left(r_{l}\right)$ with $b_{n}^{S B_{1(2)}}$ calculated by (15) and (16) in this paper; 2) $L_{\xi-W}^{T R}\left(r_{l}\right)$ with $b_{n}^{S B_{1(2)}}$ in (19) and (21) with $\beta_{T(R)}=\Delta_{H}=0$ in [8]; 3) $L_{\xi-N}^{T R}\left(r_{l}\right)$ with the numerically obtained $b_{n}^{S B_{1(2)}}$ in (15) and (16) with $\beta_{T(R)}=\Delta_{H}=0$ in [8]. The common parameters used in Figs. 2 (a) and (b) are $f_{T_{\max }}=f_{R_{\max }}=500 \mathrm{~Hz}$, $K=0, \mu_{T}^{T R}=31.2^{\circ}, k_{T}^{T R}=18.2, \mu_{R}^{T R}=216.3^{\circ}$, and $k_{R}^{T R}=10.6$. Fig. 2 (a) shows excellent agreement between the $L_{\xi-C}^{T R}\left(r_{l}\right)$ and $L_{\xi-N}^{T R}\left(r_{l}\right)$ with parameters $\Delta_{T}=\Delta_{R}=0.01$, demonstrating the correctness of our derivations.

It is worth emphasizing that the LCR, $L_{\xi-C}^{T R}\left(r_{l}\right)$, which is based on the closed-form expressions of $b_{n}^{S B_{1(2)}}$ in (15) and (16), is obtained by applying the widely used assumption $D \gg \max \left\{R_{T}, R_{R}\right\}$ in macro- and micro-cell scenarios. To fulfill this assumption, the value of parameters $\Delta_{T}$ and $\Delta_{R}$ should be chosen carefully due to the relationships $\Delta_{T} \approx$ $R_{T} / D$ and $\Delta_{R} \approx R_{R} / D$ (in general, the smaller the better).
However, the values of parameters $\Delta_{T}$ and $\Delta_{R}$ are chosen relatively large $\left(\Delta_{T}=\Delta_{R}=0.6\right)$ in [8]. This raises several questions, such as whether $\Delta_{T}=\Delta_{R}=0.6$ violates the assumption $D \gg \max \left\{R_{T}, R_{R}\right\}$, and if so, how inaccurate the LCR $L_{\xi-C}^{T R}\left(r_{l}\right)$ is, and whether this inaccuracy can be ignored. To address the above questions, we define an error function to measure the error between the LCR, $L_{\xi-G}^{T R}\left(r_{l}\right)$, with the expression of (5) based on the general expressions of parameters $b_{n}^{S B_{1(2)}}$ in (9) and (10) and $L_{\xi-C}^{T R}\left(r_{l}\right)$ as $\varepsilon=L^{-1} \sum_{l=1}^{L}\left|L_{\xi-G}^{T R}\left(r_{l}\right)-L_{\xi-C}^{T R}\left(r_{l}\right)\right| /\left|L_{\xi-G}^{T R}\left(r_{l}\right)\right|$, where $L$ is the total number of the investigated specified level $r_{l}$. Fig. 2 (b) illustrates the error $\varepsilon$ versus $\Delta\left(\Delta_{T}=\Delta_{R}=\Delta\right)$. The investigated specified level $r_{l}$ is sampled by equal-distance with number $L=200$ between $-20 \mathrm{~dB}$ and $5 \mathrm{~dB}$ in Fig. 2 (b). As expected, results show that the inaccuracy of $L_{\xi-C}^{T R}\left(r_{l}\right)$ increases with the increase of the parameter $\Delta$. From Fig. 2 (b), it is obvious that at $\Delta=0.6$, we have very high error $\varepsilon=0.894547$. This demonstrates that the chosen parameters $\Delta_{T}=\Delta_{R}=0.6$ in [8] may result in extremely inaccurate $L_{\xi-C}^{T R}\left(r_{l}\right)$. Therefore, it is desirable to propose a criterion on how to choose the parameters $\Delta_{T}$ and $\Delta_{R}$ to guarantee the accuracy of $L_{\xi-C}^{T R}\left(r_{l}\right)$ is acceptable. In this paper, we assume the accuracy of $L_{\xi-C}^{T R}\left(r_{l}\right)$ is acceptable if the error $\varepsilon \leq 0.01$. In such a case, the LCR $L_{\xi-C}^{T R}\left(r_{l}\right)$ can be applied under the condition that $\Delta_{T}=\Delta_{R} \leq 0.1085$. In other words, when $\Delta_{T}=\Delta_{R}>0.1085$ the LCR $L_{\xi-G}^{T R}\left(r_{l}\right)$ can only be used instead of $L_{\xi-C}^{T R}\left(r_{l}\right)$.

The AFD, $T_{\xi-}\left(r_{l}\right)$, is the average time over which the signal envelope, $\xi(t)$, remains below a certain level $r_{l}$. In general, the AFD $T_{\xi-}\left(r_{l}\right)$ for Ricean fading channels is defined by [10]

$$
T_{\xi-}\left(r_{l}\right)=\frac{1-Q\left(\sqrt{2 K}, \sqrt{2(K+1)} r_{l}\right)}{L_{\xi}\left(r_{l}\right)}
$$

where $Q(\cdot, \cdot)$ denotes the generalized Marcum $Q$ function.

\section{Numerical Results AND ANalysis}

In this section, we compare the theoretical results of the derived LCR and AFD in Section III with the measured data in [9]. The following parameters are used for our numerical analysis: $f_{c}=5.2 \mathrm{GHz}, f_{T_{\max }}=f_{R_{\max }}=500 \mathrm{~Hz}, \gamma_{T}=$ $\gamma_{R}=0, a=200 \mathrm{~m}, D=300 \mathrm{~m}$, and $R_{T}=R_{R}=40 \mathrm{~m}$.

Fig. 3 depicts the LCR and AFD for different outdoor picocell scenarios with parameters $\mu_{T}^{T R}=33.2^{\circ}, k_{T}^{T R}=18.2$, $\mu_{R}^{T R}=148.6^{\circ}, k_{R}^{T R}=13.3, \mu_{R}^{E R}=148.6^{\circ}$, and $k_{R}^{E R}=$ 8.6. In Figs. 3 (a) and (b), we consider the high VTD and thus assume the Ricean factor is relatively small and doublebounced rays bear more energy than single-bounced rays, i.e., $K=0.56, \eta_{D B}=0.58, \eta_{S B_{1}}=0.1, \eta_{S B_{2}}=0.18, \eta_{S B_{3}}=$ 0.14 . Figs. 3 (c) and (d) compare the theoretical LCR and AFD with the measured LCR and AFD taken from Fig. 8 (b) (highway environment with low VTD) in [9]. Therefore, we assume the Ricean factor is relatively large and single-bounced rays of the ellipse model bear more energy than single- and double-bounced rays of the two-ring model, i.e., $K=4.26$, $\eta_{D B}=0.08, \eta_{S B_{1}}=0.12, \eta_{S B_{2}}=0.18, \eta_{S B_{3}}=0.62$. 
The excellent agreement between the theoretical and measured LCR and AFD confirms the utility of the proposed model. Fig. 3 also shows that the fades are shallower when the VTD is lower, and the AFD tends to be larger with lower VTD, which meets the basic fact. This demonstrates the correctness of the method to take into account the impact of the VTD in our model.

\section{Conclusions}

In this paper, based on the developed generic model, the LCR and AFD have been derived for a non-isotropic SISO M2M Ricean fading channel. Based on the derived expressions, we have found that the VTD significantly affects the LCR and AFD of M2M channels. Finally, it has shown that the theoretical results of the LCR and AFD match very well the measured data, which validates the utility of the proposed model.

\section{ACKNOWLEDGEMENTS}

The first three authors acknowledge the support from the Scottish Funding Council for the Joint Research Institute in Signal and Image Processing between the University of Edinburgh and Heriot-Watt University which is a part of the Edinburgh Research Partnership in Engineering and Mathematics (ERPem).

\section{REFERENCES}

[1] A. S. Akki and F. Haber, "A statistical model for mobile-to-mobile land communication channel," IEEE Trans. Veh. Technol., vol. 35, no. 1, pp. 2-10, Feb. 1986

[2] A. G. Zajić and G. L. Stüber, "Space-time correlated mobile-to-mobile channels: modelling and simulation," IEEE Trans. Veh. Technol., vol. 57, no. 2, pp. 715-726, Mar. 2008.

[3] A. G. Zajić and G. L. Stüber, "Three-dimensional modeling, simulation, and capacity analysis of space-time correlated mobile-to-mobile channels," IEEE Trans. Veh. Technol., vol. 57, no. 4, pp. 2042-2054, July 2008.

[4] X. Cheng, C.-X. Wang, D. I. Laurenson, H. H. Chen, and A. V. Vasilakos, "A generic geometrical-based MIMO mobile-to-mobile channel model," IEEE IWCMC'08, Chania Crete Island, Greece, Aug. 2008, pp. 1000-1005.

[5] X. Cheng, C.-X. Wang, D. I. Laurenson, H. H. Chen, and A. V. Vasilakos, "Space-time-frequency characterization of non-isotropic MIMO mobileto-mobile multicarrier Ricean fading channels," IEEE IWCMC'08, Chania Crete Island, Greece, Aug. 2008, pp. 994-999.

[6] X. Cheng, C.-X. Wang, D. I. Laurenson, S. Salous, and A. V. Vasilakos, "An adaptive geometry-based stochastic model for MIMO mobile-tomobile channels," IEEE Trans. Wireless Commun., revised version submitted for publication.

[7] A. S. Akki, "Statistical properties of mobile-to-mobile land communication channels," IEEE Trans. Veh. Technol., vol. 43, no. 4, pp. 826-831, Nov. 1994.

[8] A. G. Zajić, G. L. Stüber, T. G. Pratt, and S. Nguyen, "Envelope level crossing rate and average fade duration in mobile-to-mobile fading channels," Proc. IEEE ICC, Beijing, China, May 2008, pp. 4446-4450.

[9] J. Maurer, T. Fügen, and W. Wisebeck, "Narrow-band measurement and analysis of the inter-vehicle transmission channel at $5.2 \mathrm{GHz}$," Proc. IEEE VTC, Birmingham, AL, USA, May 2002, pp. 1274-1278.

[10] G. L. Stüber, Principles of Mobile Communication. 2nd ed. Boston, MA: Kluwer, 2001.

[11] N. Youssef, C.-X. Wang, and M. Pätzold, "A study on the second order statistics of Nakagami-Hoyt mobile fading channels," IEEE Trans. Veh. Technol., vol. 54, no. 4, pp. 1259-1265, July 2005.

[12] M. Pätzold, U. Killat, and F. Laue, "An extended Suzuki model for land mobile satellite channels and its statistical properties," IEEE Trans. Veh. Technol., vol. 47, no. 2, pp. 617-630, May 1998

[13] A. Abdi, J. A. Barger, and M. Kaveh, "A parametric model for the distribution of the angle of arrival and the associated correlation function and power spectrum at the mobile station," IEEE Trans. Veh. Technol., vol. 51, no. 3, pp. 425-434, May 2002.
[14] I. S. Gradshteyn, and I. M. Ryzhik, Table of Integrals, Series, and Products. 5th ed, A. Jeffrey, Ed. San Diego, CA: Academic, 1994.

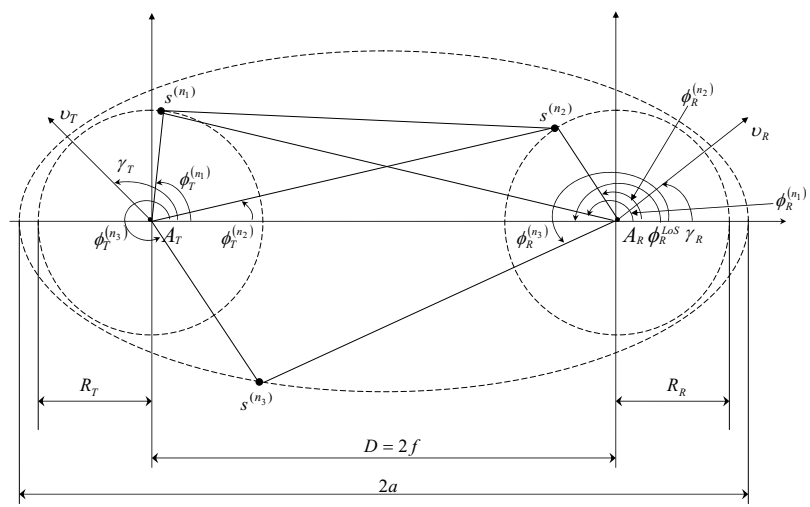

Fig. 1. The generic model for a SISO M2M channel.

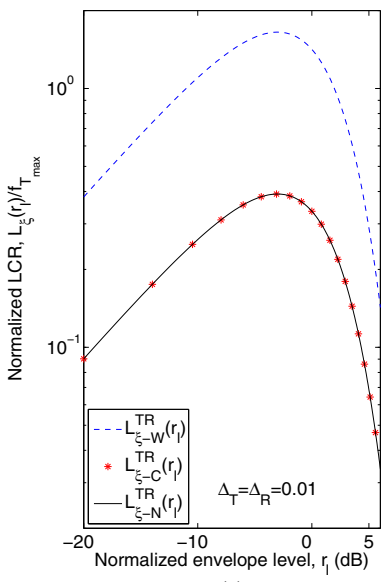

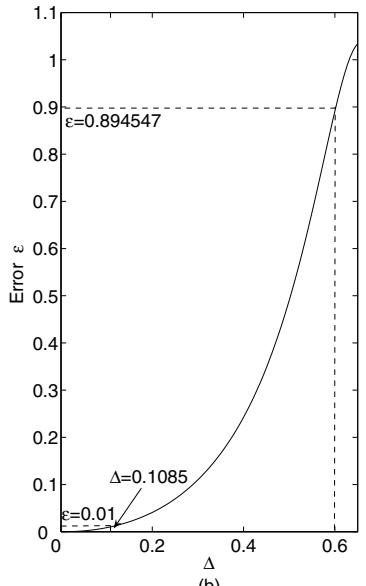

Fig. 2. (a) Comparison among the LCRs $L_{\xi-W}^{T R}\left(r_{l}\right), L_{\xi-C}^{T R}\left(r_{l}\right)$, and $L_{\xi-N}^{T R}\left(r_{l}\right)$. (b) The error $\varepsilon$ between the LCRs $L_{\xi-G}^{T R}\left(r_{l}\right)$ and $L_{\xi-C}^{T R}\left(r_{l}\right)$.
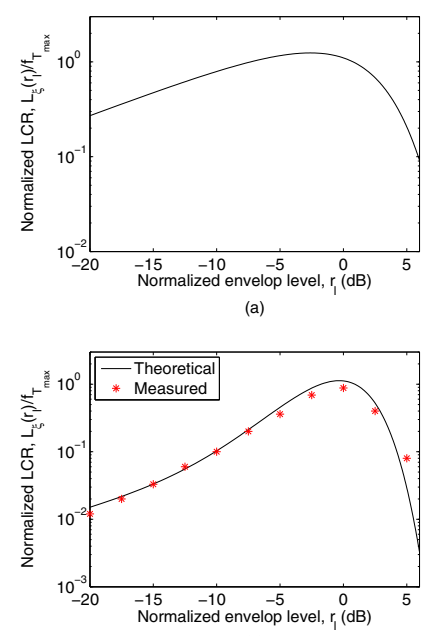
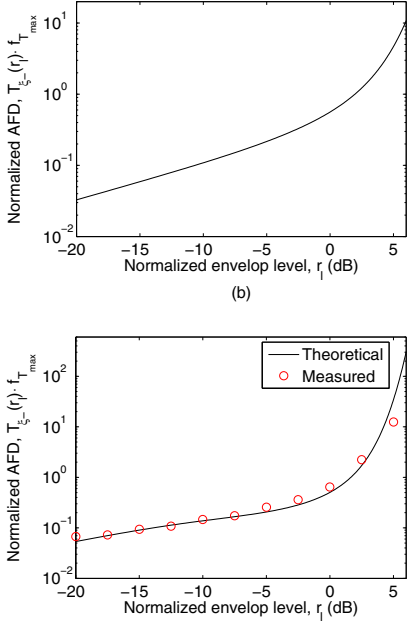

Fig. 3. The theoretical LCR (a) and AFD (b) in a pico-cell scenario with a high VTD (the Tx and Rx move in the same direction ). The theoretical and measured LCRs (c) and AFDs (d) in a pico-cell scenario with a low VTD (the Tx and Rx move in the same direction ). 